\title{
Quantifying Myocardial Oxygenation with Cardiac Magnetic Resonance Imaging
}

\author{
David J. Muccigrosso and Jie Zheng* \\ Mallinckrodt Institute of Radiology, Washington University School of Medicine, St. Louis, MO, USA
}

\begin{abstract}
Myocardial ischemia, resulting from imbalance in myocardial oxygen supply and demand, can be quantitatively assessed by positron emission tomography (PET) with absolute measures of myocardial blood flow and oxygen consumption rate $\left(\mathrm{MVO}_{2}\right)$. Cardiac Magnetic Resonance (CMR) has notable advantages over PET, with no radiation, high spatial resolution, faster scan times, and excellent soft tissue contrast. We have developed and validated new quantitative CMR oximetry techniques, including measurements of hyperemic myocardial oxygen extraction fraction and $\mathrm{MVO}_{2}$ through Fick's Law. These may lead to a new understanding of roles of myocardial microcirculation in myocardial ischemia. Other cardiac oximetry methods for directly quantifying $\mathrm{MVO}_{2}$ with ${ }^{17} \mathrm{O}$-labelled water are also under investigation. Quantitative CMR oximetry is a promising, non-invasive, non-radiation approach for exploring the myocardial metabolism's role in cardiac patients.
\end{abstract}

Keywords: Magnetic resonance imaging, ischemia, oxygen extraction, oxygen consumption.

\section{INTRODUCTION}

The heart can develop only a small oxygen debt, so oxygen supply and demand must match to maintain the normal cardiac contraction. Atherosclerotic stenosis in the macrocirculatory system (coronary arteries) causes a reduction in the blood supply. It is at the microcirculatory level that, when oxygen demand cannot be met by supply, myocardial ischemia occurs. Myocardial hypoxemia results in arrhythmia, angina, and regional or global impairment of ventricular function [1]. Severe and prolonged imbalance between oxygen supply and demand will eventually lead to myocardial infarction. In addition, ischemia may still present even though the coronary artery flow is maintained due to an imbalance between oxygen supply and demand secondary to the increased myocardial metabolic requirements. As with severe systemic hypertension, the whole heart becomes ischemic. Measuring and quantifying the balance of myocardial oxygenation would provide direct assessment of the status of myocardial oxidative metabolism and ischemic status.

In myocardial tissue, the quantitative oxygenation can be described by two parameters: myocardial oxygen extraction fraction (OEF) and oxygen consumption rate $\left(\mathrm{MVO}_{2}\right)$. Myocardial OEF reflects the fraction of oxygen extracted by myocardial tissue for oxidative metabolism, defined as $\left(\left[\mathrm{O}_{2}\right]_{\text {artery }}-\left[\mathrm{O}_{2}\right]_{\text {vein }}\right) /\left[\mathrm{O}_{2}\right]_{\text {artery }}$. $\mathrm{MVO}_{2}$ reflects the total myocardial oxygen consumption per gram of tissue. Cardiac PET remains the the gold standard for absolute quantification of regional myocardial perfusion and oxygen metabolism [2]. Currently, it is capable of accurately quantifying regional myocardial blood flow (MBF) with ${ }^{15} \mathrm{O}$-water [3-5] and $\mathrm{MVO}_{2}$ with ${ }^{11} \mathrm{C}$-acetate [6-8]. The use of ${ }^{15} \mathrm{O}_{2}$-labeled oxy-

*Address correspondence to this author at the Cardiovascular Imaging Lab, 510 South Kingshighway Blvd., Campus Box 8225, St. Louis, MO 63110 , USA; Tel: (314) 747-4608; Fax: (314) 747-3882;

E-mail: zhengj@mir.wustl.edu gen gas has also allowed absolute measurements of myocardial oxygen extraction fraction (OEF) and $\mathrm{MVO}_{2}[9,10]$. However, low spatial resolution (not suitable for the detection of subendocardial perfusion defects), ionizing radiation, limited availability, and relatively long acquisition time and high cost discourage the widespread use of PET for these purposes.

Cardiac magnetic resonance imaging (CMR) is a noninvasive imaging modality that provides excellent spatial resolution and soft tissue contrast, does not require iodinated contrast media or ionizing radiation, is widely available, and is capable of measuring cardiac function. It has been shown that CMR can measure absolute MBF and myocardial blood volume (MBV) via first-pass methods [11] and hyperemic OEF with the blood oxygen level-dependent (BOLD) technique [12]. CMR also can provide an estimation of hyperemic $\mathrm{MVO}_{2}$ through an application of Fick's Law that gives us $\mathrm{MVO}_{2} \propto \mathrm{OEF} \times \mathrm{MBF}$. These four indices of myocardial function - OEF, MBF, MBV, and $\mathrm{MVO}_{2}$ - have been the focus of our recent investigations.

Stress imaging is now widely used for the diagnosis of myocardial ischemia. Exercise or administration of the vasodilator adenosine or b-adrenergic agonist dobutamine is common mode of inducing cardiac stress. The end point is stress-induced ischemia due to a mismatch between oxygen supply and demand. On the other hand, myocardial perfusion, as quantified by MBF and blood volume (MBV), is changed during stress, either through arterial vasodilation, or secondary to the increased myocardial oxygen demand (dobutamine or exercise). All of these physiological parameters could be quantified by CMR in one imaging session.

\section{METHODS}

\section{MBF and MBV}

MBF represents myocardial perfusion and is approximately $1 \mathrm{~mL} / \mathrm{min} / \mathrm{g}$ at rest and can increase up to $4-5$ 
$\mathrm{mL} / \mathrm{min} / \mathrm{g}$ during stress. MBV at rest is approximately $6-12$ $\mathrm{mL} / \mathrm{g}$ and can increase $50-100 \%$ during stress [11]. Traditionally, MBV has been paid less attention, partially because it is less sensitive to the changes in the setting of upstream coronary artery flow and is difficult to measure accurately non-invasively. Recently, myocardial contrast echocardiography (MCE) [13] and electron-beam CT (EBCT) [14] have demonstrated proportional, but nonlinear, associations between MBV and MBF in normal animals at settings of different pharmacologically induced hyperemia. It appears that MBV plays a mediate role for the physiologic regulation of MBF and coronary blood flow. For instance, when MBF increases during adenosine injection without elevation of myocardial oxygen consumption, MBV remains constant. However, when MBF increases secondary to increases in myocardial oxygen consumption during exercise or dobutamine stress, MBV begins to increase. Accurate assessment of MBV may provide additional information about myocardial microcirculation in terms of capillary recruitment and oxygen demand [15]. At the setting of coronary artery stenosis, it was found that the magnitude of increase in $\mathrm{MBV}$ is proportional to the severity of stenosis. One could even speculated that measuring MBV heterogeneity may allow detection of coronary artery stenosis at rest without eliciting stress or vasodilation [16].

In CMR, first-pass perfusion imaging is the most accurate method for measuring MBF and MBV. While increasing efforts have been made to develop non-contrast arterial spin labeling (ASL) methods to measure MBF, MBV measurement still needs an intravascular contrast agent with this approach, as reported previously [17]. To quantify MBF \& MBV from CMR first-perfusion images, a modelindependent deconvolution algorithm (i.e., without certain modeling or assumption of vascular structure) was developed for the rapid measurement of MBF and MBV maps using blood pool contrast agents. The accuracy was validated against the comparable microsphere [18] and radio-labelled red blood cell [19] methods, respectively. In addition, a wavelet denoising algorithm was also developed to address low signal-to-noise ratio (SNR) inherent to the saturationrecovery turboFLASH sequence used in first-pass perfusion
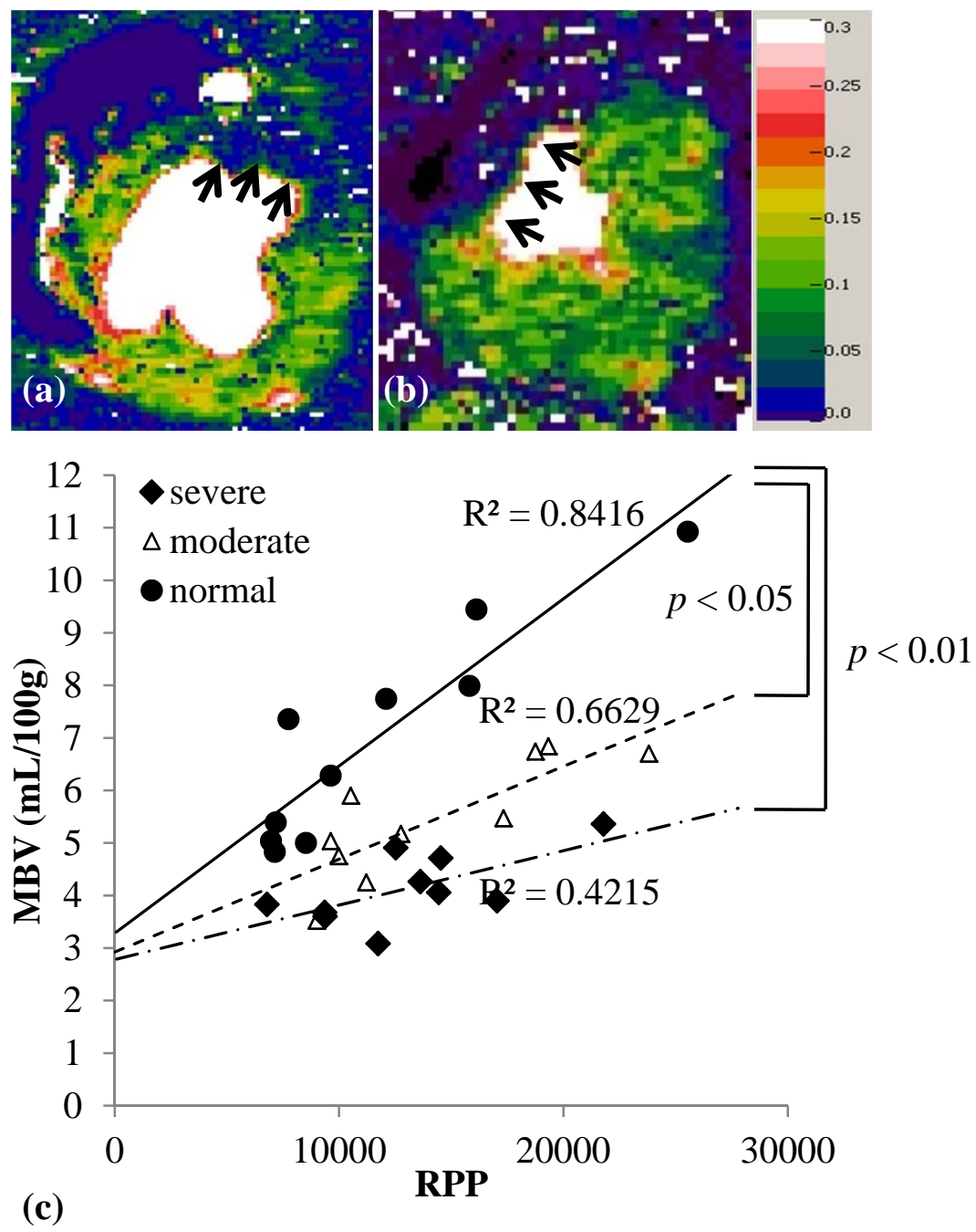

Fig. (1). Examples of MBV maps with moderate (75\% area stenosis) (a) and severe ( $95 \%$ area stenosis) (b) stenosis in anterior descending coronary arteries in dogs during dobutamine-induced hyperemia. The color scale for MBV is from 0 to $0.3 \mathrm{~mL} / \mathrm{g}$. The stenosis-subtended anterior regions had similar reduction in MBF (not shown), but MBV in the same regions reveals difference with lower intensity in map (b) (arrows). Correlation between MBV and rate-pressure product (RPP) in (c) clearly demonstrates the association of MBV with myocardial oxygen demand, which can differentiate the severities of the stenoses. 
imaging [20]. The procedure operates on both the relationships between adjacent pixels and the same pixels over time series, making it particularly useful with first-pass dynamic images.

This technical advance may allow for further exploration in the use of MRI to assess the pathophysiology of coronary artery disease and other cardiac/metabolic diseases involving impaired myocardial microcirculation. For instance, MBV was found to be more tightly associated with cardiac work than MBF, and appeared to differentiate between moderate and severe stenosis under dobutamine stress [21] (Fig. 1). In these cases, MBV increases with the elevation in oxygen demand, but acute coronary stenosis diminishes the MBV reserve. Such observation is similar as other findings that chronic infarction induced consistent elevated resting MBV and reduced $\mathrm{MBV}$ reserve in the infracted rat heart, in comparison with sham operated rat heart [22]. In addition, when measurement of $\mathrm{MVO}_{2}$ can be performed in the same imaging session of $\mathrm{CMR}, \mathrm{MBV}$ appears to have a close relationship with $\mathrm{MVO}_{2}$ when inotropic stimulation (as with dobutamine) induces relatively large changes in $\mathrm{MVO}_{2}$ [11]. In this animal study, during dipyridamole induced vasodilation, MBF reserve had a closer relationship to $\mathrm{MVO}_{2}$ reserve than $\mathrm{MBV}$ reserve. When $\mathrm{MVO}_{2}$ was more significantly altered with dobutamine induced hyperemia, MBV reserve was more closely associated with $\mathrm{MVO}_{2}$ reserve. These results are in agreement with $\mathrm{MCE}$ findings that minor increases in $\mathrm{MVO}_{2}$ can be met by increases in MBF alone; but major $\mathrm{MVO}_{2}$ increases require increases in $\mathrm{MBV}$ as well [23]. In addition to these animal studies, studies are also on the way to gain the understanding of different roles of MBV and MBF in cardiac patients. Changes in MBV appeared to be associated with the perfusion states of intermediate coronary artery stenosis [24] and the changes in $\mathrm{MVO}_{2}$ [25]. These techniques thus could provide a comprehensive evaluation of coronary stenosis severity [26].

Because no blood pool contrast agent is approved by United State Food and Drug Administration for routine CMR, measurement of MBV using blood pool agent is not practical in human. One exception is MultiHance (Bracco Diagnostic, Princeton, New Jersey), which demonstrates partial vascular retention. When one uses a small dosage of MultiHance (e.g., $0.02 \mathrm{mmol} / \mathrm{kg}$ ) for the first-pass perfusion imaging, it can be treated as a blood pool agent and the MBV obtained appears to be correct [27]. Nevertheless, systematic validation is needed and the role of MBV should be further explored in large cohorts of study in cardiac patients.

\section{OEF}

A CMR method was developed early on to derive hyperemic myocardial OEF by taking advantage of the socalled BOLD effect in myocardial $\mathrm{T}_{2}$ [12]. When myocardial flow increases during hyperemia, normal myocardial $T_{2}$ will increase secondary to reduced deoxyhemoglobin concentration. A two compartment diffusion model was generated to calculate hyperemic OEF, based on known or assumed resting OEF. The original data acquisition used a multicontrast, 2-D segmented turbo spin-echo (TSE) sequence to generate $T_{2}$-weighted images, as well as pixel-by-pixel $T_{2}$ maps.
The two-compartment approach is briefly summarized in the relationship below, without consideration of spin exchanges between intra- and extra-vascular spaces on the time scale of transverse relaxation:

$$
\begin{aligned}
& \frac{S_{\text {voxel }}(T E)}{S_{0}}=e^{-\frac{T E}{T_{2 a p p}}}=M B V \times e^{-\frac{T E}{T_{2 b}}}+(1-M B V) \times e^{-\frac{T E}{T_{2 t}}} \\
& \frac{1}{T_{2 b}}=A^{\prime} O E F^{2}+B^{\prime} O E F+C^{\prime}, \\
& \frac{1}{T_{2 t}}=R_{20 t}+R_{21 t} O E F^{2} M B V^{2} \tau^{2}
\end{aligned}
$$

where $T_{2 b}$ and $T_{2 t}$ are the $T_{2}$ values of blood and tissue, respectively. The variable $\tau$ is the inter-echo spacing in the TSE sequence. The constants $\mathrm{A}^{\prime}, \mathrm{B}^{\prime}$, and $\mathrm{C}^{\prime}$ can be derived from an established intravascular model obtained at $1.5 \mathrm{~T}$ [28], and the subject-specific parameters $R_{20 t}$ andR $R_{21 t}$ can be calculated using at least two $\mathrm{T}_{2}$ measurements at rest with different $\tau$ values [29].

In a validation study in a canine model, PET imaging was used as the gold standard to measure myocardial transmural $\mathrm{OEF}$ and $\mathrm{MVO}_{2}$. Overall there were no significant errors and the CMR oximetry results were closely correlated with gold standard PET measurements [30] (Fig. 2a). In the latest development, another $\mathrm{T}_{2}$ sequence, bright-blood $\mathrm{T}_{2}$-prepared-gradient-echo or $\mathrm{T}_{2}$-prepared TrueFISP sequence, was modified to calculate myocardial $\mathrm{T}_{2}$ maps within one breathhold. The diffusion model was simplified and the model parameters $R_{20 t}$ and $R_{21 t}$ can be obtained in one $T_{2}$ measurement at rest with up to 5 different TEs (and thus 5 different echo spacings $\tau$ ) [14]. Resting OEF in the coronary sinus, representing global myocardial OEF, can also be calculated through a blood-oxygen model. The main limitations of the sequence are relatively low spatial resolution and sensitivity to both respiratory and cardiac motion, particularly at higher heart rates. Further technical improvement can be performed in the areas of reducing motion artifacts and acquiring more TEs to increase the precision and accuracy of myocardial $\mathrm{T}_{2}$ measurements.

\section{$\mathrm{MVO}_{2}$}

Measurements of global left ventricular $\mathrm{MVO}_{2}$ and whole body oxygen consumption $\mathrm{VO}_{2}$ were reported by Yang et al. [31]. Both data are very comparable with reported PET and other invasive methods (MVO2: $11 \pm 3 \mathrm{ml} / \mathrm{min}$ per $100 \mathrm{~g} \mathrm{LV}$ mass vs. $10 \pm 3 \mathrm{ml} / \mathrm{min}$ per $100 \mathrm{~g} \mathrm{LV}$ mass; $\mathrm{VO}_{2}: 3.8 \pm 0.8$ $\mathrm{ml} / \mathrm{min} / \mathrm{kg}$ body weight vs. $3.5 \mathrm{ml} / \mathrm{min} / \mathrm{kg}$ body weight). Furthermore, the reproducibility of their measurements are relatively high (coefficient of repeatability of $1.0 \mathrm{ml} / \mathrm{min}$ per $100 \mathrm{~g} \mathrm{LV}$ mass).

To measure regional left ventricular $\mathrm{MVO}_{2}$ during hyperemia, Fick's priciple was used:

$$
M V O_{2}=1.39 \times[H b] \times O E F \times M B F \times Y_{a}
$$

where $[\mathrm{Hb}]$ is the hemoglobin concentration of the blood $(0.14 \mathrm{~g} / \mathrm{mL}), 1.39$ represents the maximum binding capacity of oxygen per unit mass of hemoglobin $(\mathrm{mL} / \mathrm{g})$, and $\mathrm{Y}_{\mathrm{a}}$ is arterial oxygen saturation (0.95). In a validation study of hyperemic $\mathrm{MVO}_{2}$ quantifications in a canine model by 


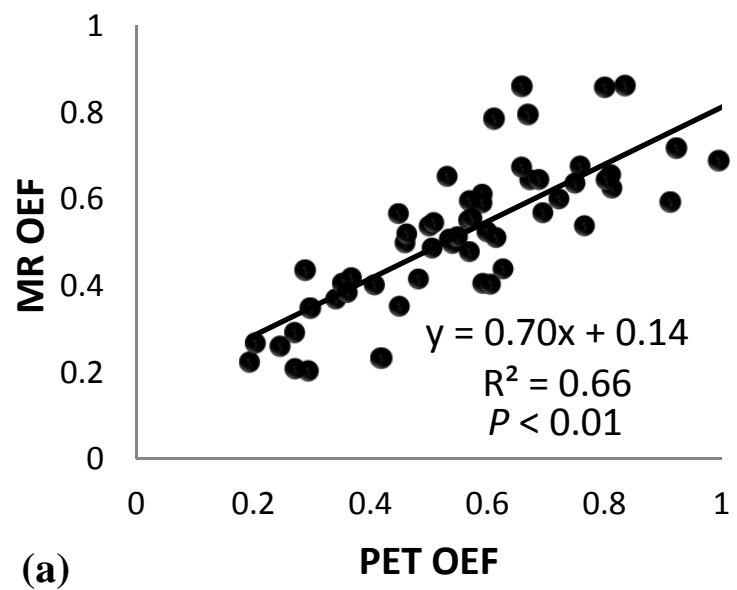

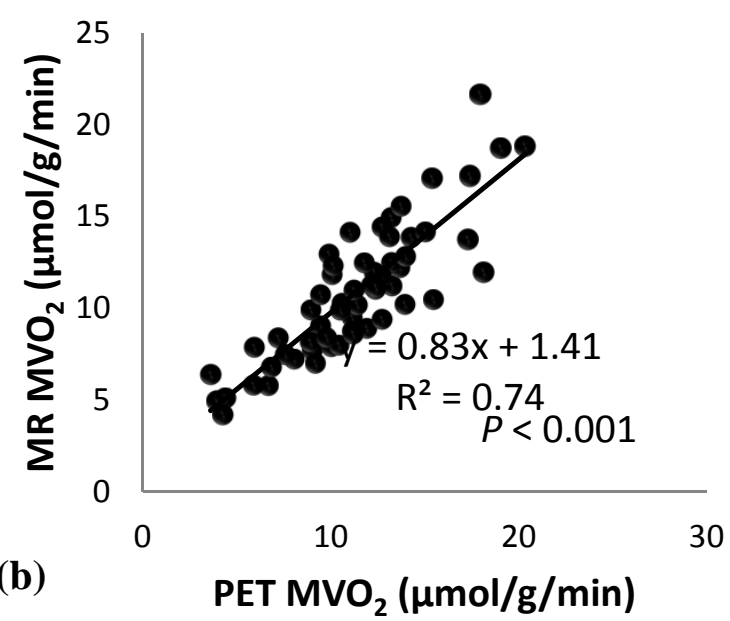

Fig. (2). Correlation of OEF and $\mathrm{MVO}_{2}$ measured with MRI and PET.

McCommis et al. [17], CMR measurements slightly overestimated PET results, but with a very strong linear correlation (slope, 0.83; intercept, 1.41; $r=0.86, P<0.001$ ) (Fig. 2b). These technical developments demonstrated promising alternatives to nuclear techniques because they could be used for serial assessments of myocardial oxygenation in settings of regional or global myocardial ischemia.

\section{CLINICAL APPLICATIONS}

\section{Coronary Artery Disease}

Current CMR methods have been developed and evaluated in canine models for the indicators of myocardial oxygen supply and demand: MBF \& MBV (supply), and OEF \& $\mathrm{MVO}_{2}$ (demand). The emerging relationship discovered in our canine model between MBV, MBF, and $\mathrm{MVO}_{2}$ suggests that, in settings of increased cardiac exertion - i.e. demand the blood flow acts as a primary reserve for normal mechanical function of the heart, while MBV serves as a secondary reserve, signifying increased capillary recruitment. This may have clinical implications in patients with myocardial ischemia, either induced by epicardial coronary artery stenosis or dysfunctional microcirculation. Furthermore, impaired myocardial perfusion is not necessary to induce myocardial ischemia if oxygen supply and demand remain balanced. In a recent publication for a clinical study in patients with coronary artery disease using oxygensensitive MRI and PET, Karamitsos et al., [32] found that $40 \%$ of myocardial segments with impaired hyperemic blood flow showed normal myocardial oxygenation at the time. Integrated CMR perfusion and oxygenation methods will likely to facilitate the comprehensive evaluation of these patients.

Another important aspect in CMR-based diagnosis is to detect subendocardial ischemia, a common form of myocardial ischemia. The subendocardium is the most at risk for ischemia because its blood, and therefore oxygen, supply has to travel the furthest. Thus, in the setting of increased oxygen demand, elevated left ventricle (LV) end-diastolic pressure and LV contraction force (e.g. exercise or dobutamine infusion), the subendocardium is the most likely to experience ischemia. Unfortunately, ischemia is not easily detected and measured in the subendocardium. The electro- cardiogram was the first noninvasive method developed with this capability, but ECG evidence of subendocardial ischemia by itself can be confused with transmural ischemia and/or electrolyte abnormalities. Real-time stress echocardiography with contrast may also detect subendocardial ischemia, but is limited in patients with poor acoustic windows (e.g., obese patients - who are often diabetic). They also do not directly measure subendocardial ischemia, but rather indirectly through its results. Thus, echo-derived measures should be less sensitive than direct ones. CMR will have the potential to directly detect altered subendocardial oxygenation. Nevertheless, diagnosis will require increasing the spatial resolution to approximately $1 \mathrm{x} 1 \mathrm{~mm}^{2}$ with adequate SNR, which should be achieved in the near future.

\section{Cardiomyopathy}

Because quantitative CMR perfusion and oxygenation imaging can potentially accurately detect myocardial ischemia, they can be used not only in diagnosis, but also to monitor the efficacy of treatment in patients with hypertensive hypertrophy, diabetic cardiomyopathy, obesity, and other form of heart failure. Zheng et al., reported impaired OEF during vasodilation in overweight and severely obese patients [33]. A linear correlation was found between hyperemic myocardial OEF and body-mass index (BMI). One important index in clinical cardiology, called cardiac efficiency, is determined by the ratio of cardiac mechanic work to $\mathrm{MVO}_{2}$. It was reported that resting $\mathrm{MVO}_{2}$ in hypertensive hypertrophy patients is similar to that in normotensive volunteers, but cardiac efficiency and myocardial blood flow reserve are significantly reduced [34, 35]. In diabetic hearts, resting $\mathrm{MVO}_{2}$ is found to be elevated with concomitant decrease in cardiac efficiency [36]. However, these studies had to use different imaging modalities (PET, ultrasound, CMR) in order to obtain the cardiac efficiency. Because CMR allows for accurate quantification of myocardial mass and mechanic work, cardiac efficiency can be quantified in the same imaging session, even in the absence of angiographically significant coronary artery stenosis. Further measurements quantifying cardiac metabolism in patients prone to heart failure and a variety of cardiomyopathy may lead to the development of new protocols which elucidate microcirculatory dysfunction and its related cardiac remodeling. 


\section{Other Methodology for Quantifying $\mathrm{MVO}_{2}$}

Despite successful quantitative assessment of regional myocardial oxygenation during hyperemia, regional resting oxygenation cannot be measured with the BOLD effect. Another new technology was recently developed by Zheng et al., [37] to introduce ${ }^{17} \mathrm{O}$-labelled blood as a contrast agent to quantify myocardial oxygenation. The potential advantage of this new method is that we can directly monitor regional myocardial oxygenation at rest or during hyperemia. Once the ${ }^{17} \mathrm{O}$-labelled agent enters the blood system, it is converted metabolically to $\mathrm{H}_{2}{ }^{17} \mathrm{O}$ in the mitochondria and then washed out from the venules into circulating blood. This may allow for indirect measurements of tissue $\mathrm{H}_{2}{ }^{17} \mathrm{O}$ concentration and oxygen consumption rate. This quantification method relies on the image contrast obtained from a spinlocking $\mathrm{T}_{1} \rho$-weighted imaging sequence. The $\mathrm{H}_{2}{ }^{17} \mathrm{O}$ concentration can be calculated using a technique established in cerebral applications [38]. However, challenges remain to accurately quantify $\mathrm{MVO}_{2}$ using the ${ }^{17} \mathrm{O}$-labelled contrast agent method: 1) sensitivity of the contrast detection is relatively low (10 - 15\% signal changes), particularly when the heart motion is not controlled well; 2) a comprehensive model has yet to be established for this intravenous injection method, although quantitative models for the inhalation methods in brain studies were reported previously [39, 40].

\section{CONCLUSION}

CMR oximetry has demonstrated the capability to accurately quantify myocardial oxygenation imaging. The image-based quantification of all four indices of myocardial supply and demand has been validated against respective gold standard methods. Further research to improve our techniques is under consideration, including using alternative imaging sequences (such as Cartesian or radial true-FISP) for better SNR, minimizing cardiac motion (navigator-echo gating or self-gating), and establishing a computational model for ${ }^{17} \mathrm{O}$-based calculation. The lack of capability to measure regional resting OEF is also of concern, although the newer ${ }^{17} \mathrm{O}$ method may meet this requirement. The ultimate goal would be for CMR oximetry and perfusion quantification methods to integrate as a fast, convenient modality for quantifying myocardial oxygen supply and demand.

As many CMR methods improve, studies in normal and cardiac dysfunctional patients may help us to elucidate the mechanism linked with myocardial metabolism and remodeling. This avenue of research will eventually allow us to identify links between various conditions and the balance of myocardial oxygen supply and demand, building an effective tool in cardiac diagnostics and treatment monitoring.

\section{CONFLICT OF INTEREST}

None declared.

\section{ACKNOWLEDGEMENT}

This work was supported by a grant from the National Institutes of Health R01 HL74019 and R21 EB009125.

\section{REFERENCES}

[1] Braunwald E. Heart disease. $6^{\text {th }}$ ed. Philadelphia: W.B. Saunders Company 2001.

[2] Segall G. Assessment of myocardial viability by positron emission tomography. Nucl Med Commun 2002; 23: 323-30.

[3] Bergmann SR, Herrero P, Markham J, Weinheimer CJ, Walsh MN Noninvasive quantitation of myocardial blood flow in human subjects with oxygen-15-labelled water and positron emission tomography. J Am Coll Cardiol 1989; 14: 639-52.

[4] Gropler RJ, Geltman EM, Sampathkumaran KS. Comparison of C11 acetate with F-18 fluorodeoxyglucose for delineating viable myocardium by positron emission tomography. J Am Coll Cardiol 1993; 23: 1587-97.

[5] Sun KT, Yeatman LA, Buxton DB, et al. Simultaneous measurement of myocardial oxygen consumption and blood flow using [1-carbon-11] acetate. J Nucl Med 1998; 39: 272-80.

[6] Brown MA, Myears DW, Bergmann SR. Validity of estimates of myocardial oxidative metabolism with carbon-11 acetate in positron emission tomography despite altered patterns of substrate utilization. J Nucl Med 1989; 30: 187-93.

[7] Yamamoto $\mathrm{Y}$, de Silva R, Rhodes CG, et al. Noninvasive quantification of regional myocardial metabolic rate of oxygen by $15 \mathrm{O} 2$ inhalation and positron emission tomography. Experimental validation. Circulation 1996; 94: 808-16.

[8] Gropler RJ, Geltman EM, Sampathkumaran KS. Functional recovery after revascularization for chronic coronary artery disease is dependent on maintenance of oxidative metabolism. J Am Coll Cardiol 1992; 20: 569-77.

[9] Iida H, Rhodes CG, Araujo LI, et al. Noninvasive quantification of regional myocardial metabolic rate for oxygen by use of $15 \mathrm{O} 2$ inhalation and positron emission tomography. Theory, error analysis, and application in humans. Circulation 1996; 94: 792-807.

[10] Agostini D, Iida H, Takahashi A, Tamura Y, Henry Amar M, Ono $\mathrm{Y}$. Regional myocardial metabolic rate of oxygen measured by $\mathrm{O} 2$ 15 inhalation and positron emission tomography in patients with cardiomyopathy. Clin Nucl Med 2001; 26: 41-9.

[11] McCommis KS, Zhang HS, Goldstein TA, et al. Myocardial Blood Volume Is Associated with Myocardial Oxygen Consumption: An Experimental Study with CMR in a Canine Model. JACC Cardiovasc Imag 2009; 2: 1313-20.

[12] Zheng J, Wang JH, Nolte M, Li D, Gropler RJ, Woodard PK. Dynamic estimation of myocardial oxygen extraction ratio during dipyridamole stress by MRI: A preliminary study in canines. Magn Reson Med 2004; 51: 718-26.

[13] Le DE, Bin JP, Coggins MP, Wei K, Lindner JR, Kaul S. Relation between myocardial oxygen consumption and myocardial blood volume: a study using myocardial contrast echocardiography. J Am Soc Echocardiogr 2002; 15: 857-63.

[14] Möhlenkamp S, Lerman LO, Lerman A, et al. Minimally invasive evaluation of coronary microvascular function by electron beam computed tomography. Circulation 2000; 102: 2411-6.

[15] Wei K, Kaul S. The coronary microcirculation in health and disease. Cardiol Clin 2004; 22: 221-31.

[16] Wu CC, Feldman MD, Mills JD, et al. Myocardial contrast echocardiography can be used to quantify intramyocardial blood volume: new insights into structural mechanisms of coronary autoregulation. Circulation 1997; 96: 1004-11.

[17] Waller C, Kahler E, Hiller KH, et al. Myocardial perfusion and intercapillary blood volume in rats at rest and with coronary dilatation: MR imaging in vivo with use of a spin-labeling technique. Radiology 2000; 215: 189-97.

[18] Goldstein TA, Jeorsch-Herold M, Misselwitz B, Zhang HS, Gropler RJ, Zheng J. Fast mapping of myocardial blood flow with MR firstpass perfusion imaging. Magn Reson Med 2008; 59: 1394-400.

[19] McCommis K, Goldstein TA, Zhang H, Misselwitz B, Gropler RJ, Zheng J. Quantification of myocardial blood volume during dipyridamole and dobutamine stress: a perfusion MRI study. J Cardiovasc Magn Reson 2007; 9: 785-92.

[20] Goldstein TA, Zhang H, Misselwitz B, Gropler RJ, Zheng J. Improvement of quantification of myocardial first-pass perfusion mapping: a temporal and spatial wavelet denoising method. Magn Reson Med 2005; 56: 439-45.

[21] McCommis KS, Goldstein TA, Abendschein D, et al. Roles of myocardial blood volume and flow in coronary artery disease: an experimental MRI study at rest and during hyperemia. Eur Radiol 2010; 20: 2005-12. 
[22] Waller C, Hiller KH, Kahler E, et al. Serial magnetic resonance imaging of microvascular remodeling in the infarcted rat heart. Circulation 2001; 103: 1564-9.

[23] Bin JP, Pelberg RA, Wei K, Le E, Goodman NC, Kaul S. Dobutamine versus Dipyridamole for inducing reversible perfusion defects in chronic multivessel coronary artery stenosis. J Am Coll Cardiol 2002; 40: 167-74.

[24] Firschke C, Andrássy P, Linka AZ, Busch R, Martinoff S. Adenosine myocardial contrast echo in intermediate severity coronary stenoses: a prospective two-center study. Int J Cardiovasc Imaging 2007; 23: 311-21.

[25] Bin JP, Le DE, Jayaweera AR, Coggins MP, Wei K, Kaul S. Direct effects of dobutamine on the coronary microcirculation: comparison with adenosine using myocardial contrast echocardiography. J Am Soc Echocardiogr 2003; 16: 871-9.

[26] Lindner JR, Skyba DM, Goodman NC, Jayaweera AR, Kaul S. Changes in myocardial blood volume with graded coronary stenosis. Am J Physiol 1997; 272: H567-75.

[27] McCommis KS, O`Connor R, Donna L, Woodard PK, Gropler RJ, Zheng J. Quantification of global and regional myocardial oxygenation in humans: initial experiences. J Cardiovasc Magn Reson 2010; 12: 34.

[28] Golay X, Silvennoinen MJ, Zhou J, et al. Measurement of tissue oxygen extraction ratios from venous blood $\mathrm{T}(2)$ : increased precision and validation of principle. Magn Reson Med 2001; 46: $282-91$.

[29] Zheng J, Wang JH, Rowold FE, Gropler RJ, Woodard PK. Relationship of apparent myocardial T2 and oxygenation: towards quantification of myocardial oxygen extraction fraction. J Magn Reson Imaging 2004; 20: 233-41.

[30] McCommis KS, Goldstein TA, Abendschein DR, et al. Quantification of regional myocardial oxygenation by magnetic resonance imaging: validation with positron emission tomography. Circ Cardiovasc Imag 2010; 3: 41-6.

[31] Yang Y, Foltz WD, Merchant N, Stainsby JA, Wright GA. Noninvasive quantitative measurement of myocardial and whole- body oxygen consumption using MRI: initial results. Magn Reson Imag 2009; 27: 147-54.

[32] Karamitsos TD, Leccisotti L, Arnold JR, et al. Relationship between regional myocardial oxygenation and perfusion in patients with coronary artery disease: insights from cardiovascular magnetic resonance and positron emission tomography. Circ Cardiovasc Imag 2010; 3: 32-40.

[33] Zheng J, Lesniak D, O'Connor R, et al. Assessment and validation of cardiac MR oximetry in obesity. Proc Intl Soc Mag Reson Med; Stockholm, Sweden 2010; p. 484.

[34] Laine H, Katoh C, Luotolahti M, et al. Myocardial oxygen consumption is unchanged but efficiency is reduced in patients with essential hypertension and left ventricular hypertrophy. Circulation 1999; 100: 2425-30.

[35] de las Fuentes L, Soto PF, Cupps BP, et al. Hypertensive left ventricular hypertrophy is associated with abnormal myocardial fatty acid metabolism and myocardial efficiency. J Nucl Cardiol 2006; 13: 369-77.

[36] How OJ, Aasum E, Severson DL, et al. Increased myocardial oxygen consumption reduces cardiac efficiency in diabetic mice. Diabetes 2006; 55: 466-73.

[37] McCommis KS, He X, Abendschein D, Gupte PM, Gropler RJ, Zheng J. Cardiac ${ }^{17} \mathrm{O}$ MRI: Towards direct quantification of myocardial oxygen consumption. Magn Reson Med 2010; 63: $1442-7$.

[38] Tailor DR, Baumgardner JE, Regatte RR, Leigh JS, Reddy R. Proton MRI of metabolically produced $\mathrm{H}_{2} 17 \mathrm{O}$ using an efficient $17 \mathrm{O}_{2}$ delivery system. Neuroimage $2004 ; 22: 611-8$.

[39] Mellon EA, Beesam RS, Baumgardner JE, Borthakur A, Witschey WR 2nd, Reddy R. Estimation of the regional cerebral metabolic rate of oxygen consumption with proton detected $17 \mathrm{O}$ MRI during precision $17 \mathrm{O}_{2}$ inhalation in swine. J Neurosci Methods 2009; 179: 29-39.

[40] Atkinson IC, Thulborn KR. Feasibility of mapping the tissue mass corrected bioscale of cerebral metabolic rate of oxygen consumption using 17-oxygen and 23-sodium MR imaging in a human brain at 9.4 T. Neuroimage 2010; 51: 723-33. 